\title{
ELP-OA: status report of the setup of the demonstrator of the Polychromatic Laser Guide Star at Observatoire de Haute-Provence
}

\author{
Renaud Foy ${ }^{1,2}$, Nicolas Meilard ${ }^{1}$, Michel Tallon ${ }^{1}$, Éric Thiébaut ${ }^{1}$, Pierre-Éric Blanc ${ }^{2}$, Michel Boër $^{2}$, \\ Julien Chombart ${ }^{2}$, Andrée Laloge ${ }^{2}$,Auguste Le Van $\mathrm{Suu}^{2}$, Sandrine Perruchot ${ }^{2}$, Xavier Régal ${ }^{2}$, \\ Jérôme Schmitt ${ }^{2}$, Alain Petit ${ }^{3}$, Thierry Fusco ${ }^{4}$ \\ 1 Université de Lyon, Lyon, F-69003, France ; Université Lyon 1, Observatoire de Lyon, 9 avenue \\ Charles André, Saint-Genis Laval, F-69230, France ; CNRS, UMR 5574, Centre de Recherche As- \\ trophysique de Lyon ; Ecole Normale Supérieure de Lyon, Lyon, F-69007, France \\ 2 Observatoire de Haute-Provence, 04870 Saint-Michel l'Observatoire, France ; CNRS, USR 2207 \\ 3 DEN/DANS/DPC, Commissariat à l'Énergie Atomique Saclay, 91191 Gif-sur-Yvette cedex, France \\ 4 ONERA, BP. 52, 29 avenue de la Division Leclerc, F-92320 Chatillon cedex, France
}

\begin{abstract}
We report the status of ELP-OA (Étoile Laser polychromatique pour l'Optique Adaptative), the full demonstrator which we are building at OHP $1.52 \mathrm{~m}$ telescope. The goal is to open adaptive optics to the domain of visible wavelengths at large telescopes, which is almost not feasible today because of the tiny isoplanatic patch. ELP-OA relies on the 2-photon excitation of sodium in the mesosphere, through 589 and $569 \mathrm{~nm}$ transitions. We use 2 pulsed dye lasers (on loan from CEA) pumped with NdYAGs. The average power at the mesosphere will be $2 \times 22 \mathrm{~W}$. The twin laser beams are projected to the mesosphere by a 3 -aperture interferometer. The backscattered spots in NaI lines at 330, 569 and 589nm are oberved through an adaptive optics at the telescope coudé focus, derived from the ONERA's BOA device. The differential tilt measurement channel is equipped with an EMCCD. We use a correlation algorithm to extract it. From our end to end model we expect tilt Strehl ratios of $35 \%$ at $550 \mathrm{~nm}$ (see Meilard et al in this conference). First lasers launches are planned early 2010, with the full experiment running 2011.
\end{abstract}

\section{Introduction}

Adaptive optics (AO) requires at least one source of reference bright enough within the isoplanatic patch of the source of interest, to provide us with diffraction limited long exposure images at large telescopes. When a natural reference is lacking, In case the wavefront phase there is no such an object for phase reference, it can be measured thanks to a laser guide star [1] (LGS). LGS's generally rely on the transition between the two first energy levels $3 S_{1 / 2} \rightleftarrows 3 P_{3 / 2}$ of sodium atoms in the mesosphere (the $D_{2}$ line). Because of the tilt or tilts between the emitting laser and the receiving wavefront sensor, the location of the laser spot in the mesosphere is unknown[2].

The first way to overcome this problem is to measure the tilt from a natural guide star (NGS); for the tilt order, its is right to speak about a "guide" star, whereas strictly speaking for higher order one should more to speak about "phase reference star". But the tip tilt anisoplanatic patch is again tiny at visible wavelengths : a few arc seconds, so that sky coverage is dramatically low, much less than $1 \%$.

ELTs need LGS aided AO. Table 1 summarizes some of the astrophysical programs proposed for the E-ELT in workshops help in Florence (2004) and Vienna (2004). All these programs in the visible and some at IR wavelengths will definitely require that AOs sense the tilt from the laser star itself. Most often, it cannot be with the current LGSs.

The ELP-OA (Étoile Laser polychromatique pour l'Optique Adaptative) aims at break this lock for visible wavelengths. We are conducting at Observatoire de Haute-Provence (OHP, Southern France). ELP-OA is not an instrument, but an experiment to prove that the measurement of the wavefront tilt without NGS is possible from the only polychromatic LGS (PLS).

The PLGS concept relies on the variation of the air refraction index $n$ with $\lambda$, mostly in the UV. It causes a slight variation of the tilt $\theta \lambda$. Thanks to the principle of variable separability [3]:

This is an Open Access article distributed under the terms of the Creative Commons Attribution-Noncommercial License, which permits unrestricted use, distribution, and reproduction in any noncommercial medium, provided the original work is properly cited. 
First conference on Adaptive Optics for Extremely Large Telescopes

\begin{tabular}{|c|c|c|}
\hline Topics & $\begin{array}{l}\text { Spatial } \\
\text { resolution }\end{array}$ & $\begin{array}{l}\text { Spectral } \\
\text { range }\end{array}$ \\
\hline PLANETS & & \\
\hline Exoplanets & $1-2$ mas & $0.6-1.4 \mu \mathrm{m}$ \\
\hline Solar System & 5mas@1 @1 m & vis - thermal IR \\
\hline STARS AND DISKS & & \\
\hline Probing birth places & 2-10mas@ NIR & $1-5 \mu \mathrm{m}$ \\
\hline Normal and peculiar stars & 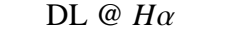 & $H \alpha$ \\
\hline Chemical comp.: chronometry & "high" & $300-700 \mathrm{~nm}$ \\
\hline $\mathrm{BHs}$ in GCs & $\approx 1 \mathrm{mas}$ & $\mathrm{K}$, CaII, vis. \\
\hline STARS \& GALAXIES & & \\
\hline Resolved Stellar Populations & DL & $V-K$ \\
\hline Resolved Stars in Clusters & 3 mas & $400-600 \mathrm{~nm}$ \\
\hline Stellar Kinematic Archaeology & DL & $\mathrm{V}-\mathrm{K}$ \\
\hline Intracluster Stellar Population & DL & $\mathrm{J} \& \mathrm{~K}$ \\
\hline Cosmic SFR from SNs & $\mathcal{S} \approx 0.5$ & $\mathrm{~J}-\mathrm{H}-\mathrm{K}$ \\
\hline Young, Massive Star Clusters & $30-40$ mas & $>0.8 \mu \mathrm{m}$ \\
\hline Black holes in GN & $\mathrm{DL} \lesssim 1 \mu \mathrm{m}$ & Visible \& NIR \\
\hline
\end{tabular}

Table 1. Programmes at ELTs requiring a Polychromatic LGS (source: Florence \& Vienna workshops)

$$
n(\lambda, P, T)-1=f(\lambda) \times g(P, T)
$$

where $P$ and $T$ stand for the atmospheric pressure and temperature. Derivating Eq. 1 yields:

$$
\Delta n /(n-1)=\Delta f(\lambda) / f(\lambda)
$$

When we apply Eq.2 to the tilt order $\theta$ of the wavefront expansion (piston being removed), we get:

$$
\theta_{\lambda_{3}}=\Delta \theta_{\lambda_{1}, \lambda_{2}}\left(n_{\lambda_{3}}-1\right) / \Delta n_{\lambda_{1}, \lambda_{2}} .
$$

Thus the tilt $\theta$ at $\lambda_{3}$ can be derived from $\Delta \theta$ between $\lambda_{1}$ and $\lambda_{2}$ (but $\lambda_{3}$ can be one of $\lambda_{1}$ or $\lambda_{2}$ as well). The larger is the wavelength difference and the shorter is the shortest wavelength, the higher is the differential tilt and accordingly the sensitivity.

The PLGS has to emit at two $\lambda$ s at least, one of them in the UV. We produce it with a 2-photon excitation of the $4 D_{5 / 2}$ level of $\mathrm{Na}$ in the mesosphere, via the $3 P_{3 / 2}$ one. The spectrum of the radiative decay from the $4 D_{5 / 2}$ level extends from $0.33 \mu \mathrm{m}$ up to $2.34 \mu \mathrm{m}$. Using these $\lambda$ s leads to $\theta / \Delta \theta=18$.

\section{Laser-sodium interaction}

We have modeled the laser-sodium interaction in order to constrain and to optimize as far as possible the free parameters of the laser chains. We have used the BEACON code developed at CEA. BEACON is based on the optical Bloch formalism [4], where the atom follows a quantum model and the laser field a classical one. BEACON computes the density matrix evolution as a function of the hamiltonian of the isolated atom + a function of the hamiltonian of $\mu \vec{E}$, where $\vec{E}$ is the laser electric field. Rate equation models are not suitable for this excitation mechanism, since $\frac{\partial \vec{E}}{\partial t} \neq 0$, both because of energy level lifetimes and of the frequency of the electric field modulation function used to broaden the laser spectrum.

The main free input parameters in BEACON are summarizes here, with typical values:

- laser power density $p\left(\mathrm{~W} / \mathrm{cm}^{2}\right)$ has to take into account the average power $2 \times 22 \mathrm{~W}$

- pulse time profile , gaussian

- pulse FWHM $\Delta t$ variable, $40 \mathrm{~ns}$ 


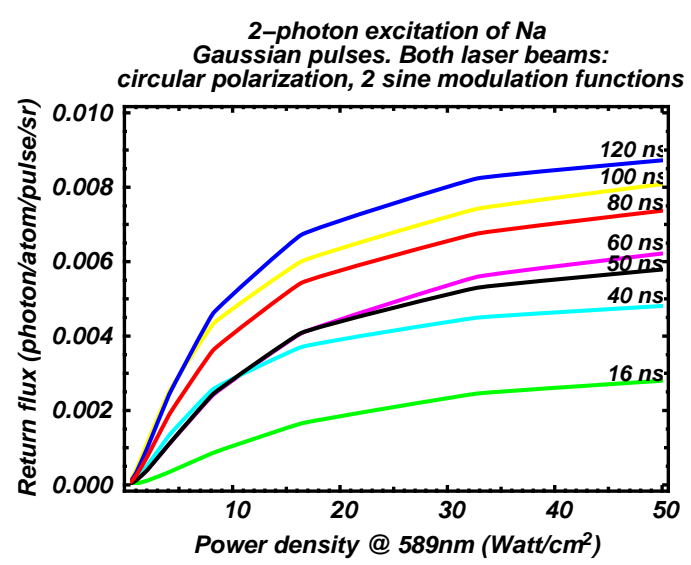

Fig. 1. Scattered flux at $330 \mathrm{~nm}$ per atom per laser pulse per steradian versus the pulse length, for a power density of $1 \mathrm{~W} / \mathrm{cm}^{2}$, i.e below saturation becomes significant.

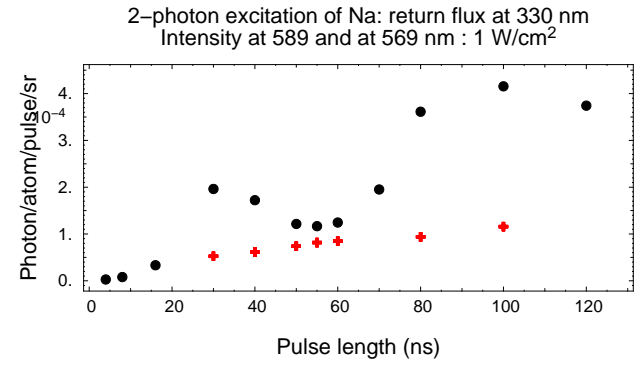

Fig. 2. Backscattered flux at $330 \mathrm{~nm}$ versus pulse length. Black dots: an optimized phase modulation fuction. Red crosses: modulation used for PASS-2 experiment at CEA/Pierrelatte.

- delay $\delta t$ between pulses at 589 and at $569 \mathrm{~nm}$, a few ns

- time to reach maximum peak power : $2 \delta t$ is conservative

- spectral shift used to optimize the modulation function

- phase modulation parameters number of functions, amplitude and frequency

Figure 1 shows the return flux versus $p$ for a set of pulse FWHM lengths. One could deduce from it that the longest the pulse the highest the return flux. But increasing $p$ at constant pulse length and repetition rate increases $\bar{P}$, which does not fit experimental conditions! Such graphs are used to compute the return flux spatial distribution in the mesosphere at given $\bar{P}$ for different laser intensity distributions. The maximum return flux is obtained for $\approx 40 \mathrm{~ns}$.

Figure 2 shows the return flux at $330 \mathrm{~nm}$ versus the pulse length for two modulation functions. Crosses refer to the function used for our PASS-2 experiment at CEA/Pierrelatte [5], and filled circles to one of the best twin sine functions for each of the two laser beams at 589 and $569 \mathrm{~nm}$. Near 40ns, a gain by a factor of $\approx 3$ is expected with the new modulation function.

\section{The interferometric projector}

Since the PLGS relies on a differential effect, a major question arises : does it return enough flux to allow us to measure $\Delta \theta$ and to compute $\theta$ with an accuracy to the level required by diffraction limited imaging in the visible?

Basically, this accuracy is driven by the ratio

$$
\sigma(\theta) \propto F W H M / \sqrt{N} .
$$

With a classical projector, the answer is no : either the predicted return flux at $330 \mathrm{~nm}$ is too low or/and the FWHM of the spot is too large. Increasing the diameter produces speckle noise. If AO precompensates for the upward beam, saturation of $\mathrm{Na}$ absorption will occur, so that there is no benefit. Since we cannot increase the return flux up to a point, we do need to increase the projector without introducing speckle noise. And this is where interferometry now enters the picture, to lead to an Interferometric PLGS (IPLGS)

Following the Cramér-Rao criterion, $\sigma(\theta)$ is limited not by the $F W H M$ but by the smallest resolved features in the laser spot image, as the interferogram produced in the mesosphere by e.g. a 3 aperture interferometer. From these simple statements, we have derived the specifications for the IPLGS projector : 


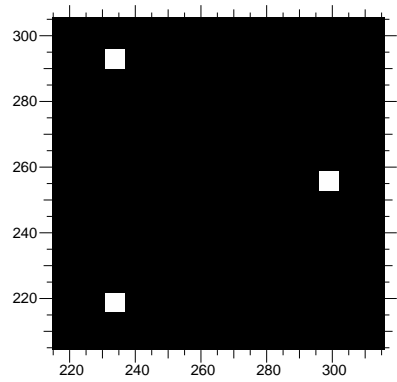

Fig. 3. Pupil configuration of the array

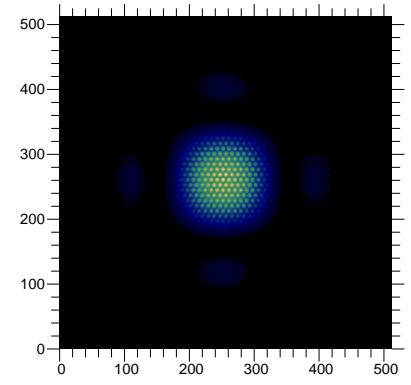

Fig. 4. Image of the interferogram at the master telescope $+\mathrm{OA}$

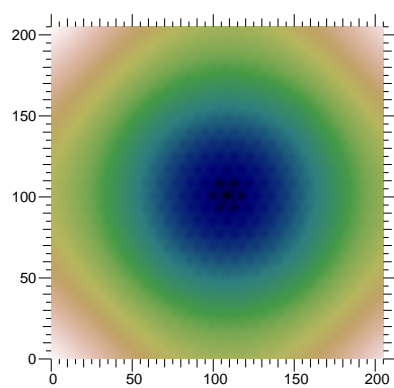

Fig. 5. Map of the criterion minimizing the distance between the model and observed interferograms
ELP-OA interferometric projector $\left\langle P_{589}\right\rangle=\left\langle P_{569}\right\rangle=22 \mathrm{~W}$ at the mesosphere

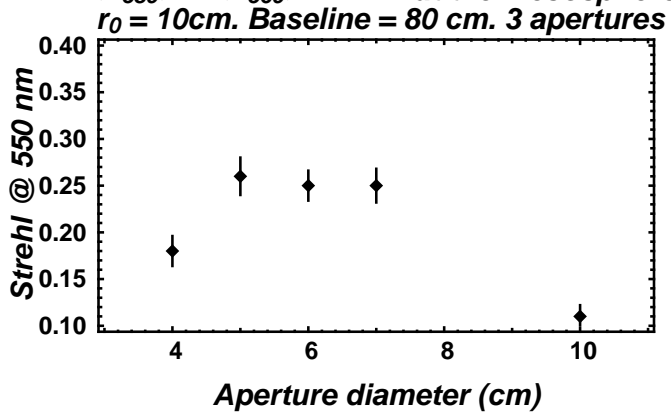

Fig. 6. An optimization of the single aperture diameter

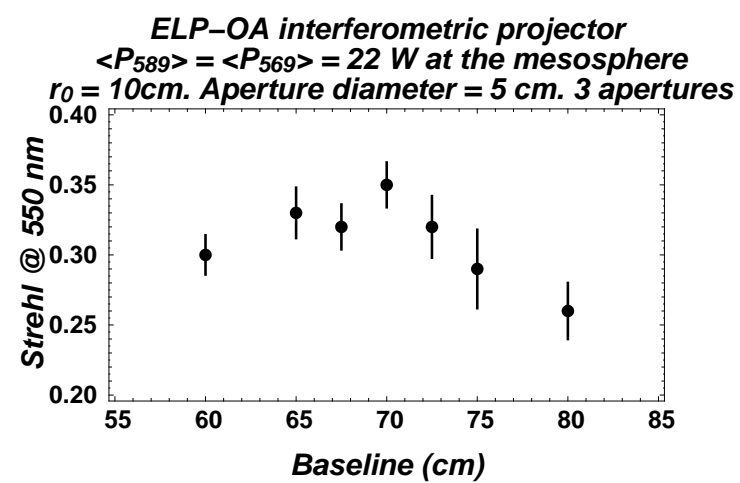

Fig. 7. An optimization of the baseline

- the fringe period has to be $>\lambda / D \approx 77$ mas, to avoid a strong interferometric projector attenuation of the visibility due to the $1.5 \mathrm{~m}$ telescope MTF, where $D$ is the reception telescope diameter

- no speckle lead to the single aperture diameter $d$ of single apertures of the array such as $d / r_{0} \lesssim 1$

- the pattern has to be translation invariant ; thus there must be at most 1 phase closure $\Longrightarrow$ at most 3 apertures. We note that a configuration 2 twin telescope interferometers with orthogonal baselines also meet this requirement

- the IPLGS needs to be resolved by the master telescope so that it has to equipped with an AO, running down to $330 \mathrm{~nm}$

- on line data processing should not rely on a center of gravity algorithm which would smear out the interfence pattern. Instead we are using 2D (spatial) cross correlations between a model interferogram with each of the 2 interferograms at 569 and $330 \mathrm{~nm}$; we set the constrain of invariance by translation in order to be able to use a model. We are developing 3D correlations (1D time $+2 \mathrm{D}$ space) to further improve the accuracy in $\Delta \theta$ measurements.

Figure 4 show the perfect interferogram observed at the master telescope (without turbulence perturbing the return beam) for the array configuration in Fig. 3. Figure 5 shows the map of the values of the criterion minimizing the distance between the observed interferogram and the model, weighted by the Poisson noise of the photon distribution (see Meilard et al paper in this proceedings).

Fig. 7 and 6 shows an optimization of the single aperture diameter of the projector, and of its baseline $B$. We adopt $d \approx 5 \mathrm{~cm}$ and $B \approx 70 \mathrm{~cm}$ (see details in Meilard et al paper in this proceedings). 


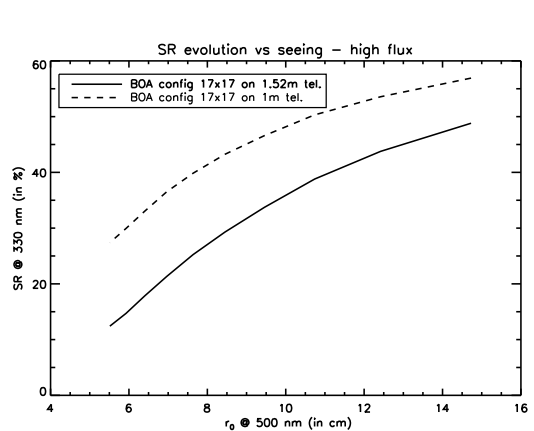

Fig. 8. Expected performances of a $17^{2}$ actuators adaptive optics device at an $1.52 \mathrm{~m}$ telescope at $330 \mathrm{~nm}$. Case of a bright reference source.
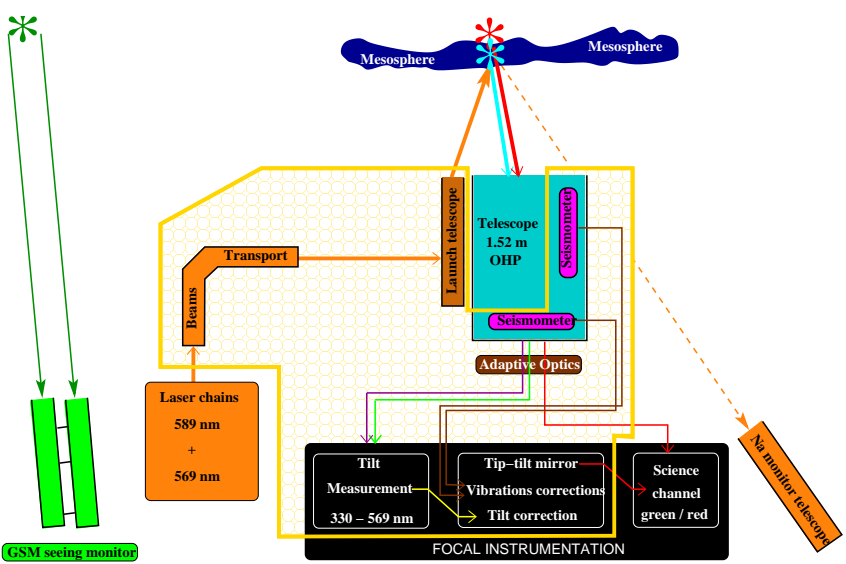

Fig. 9. Layout of the ELP-OA experiment at the $1.52 \mathrm{~m}$ telescope at OHP.

\section{Adaptive optics}

The AO at the coudé focus of the $1.52 \mathrm{~m}$ telescope will be BOA-UV, directly derived from the ONERA's BOA device[6]. The main modification required specifically for ELP-OA comes from the specification to have the best as possible transmission at $330 \mathrm{~nm}$. It concerns the focal adapter at the entrance of the bench. The remaining optics is catadioptric.

Another change, also recently done at ONERA, is required to allow BOA to fit with the ALPAO $17^{2}$ actuators deformable mirror, which is smaller than the original DM. Finally attention has to paid to the field of the wavefront sensor, fed by the $589 \mathrm{~nm}$ return light, and no longer by a natural star.

It is often claimed that OA cannot work down to $330 \mathrm{~nm}$, a very short wavelength indeed. Model performances for this device are shown in Fig. 8. If necessary we will diaphragm the telescope aperture down to $1 \mathrm{~m}$.

\section{End-to-end model}

We have developed a model of the whole PLGS process, from the output of the laser chains to the tilt Strehl ratio obtained at the focus of BOA-UV (for details, see Meilard et al paper in this proceedings). In summary, our model include the following steps :

- Lasers $\bar{P}, F_{R}$, modulation, polarization ...

- Projector pupil spatial distribution, transmission $(\approx 80 \%)$

- Atmosphere (up-link) Kolmogorov phase screen $\left(5 \leq r_{0} \leq 10 \mathrm{~cm}\right)$, transmission $(80 \%)$

- Mesosphere Na column density $\left(4 \times 10^{13}\right.$ atoms $\left./ \mathrm{m}^{2}\right)$, altitude $(92 \mathrm{~km})$

- Atmosphere (down-link) same Kolmogorov phase screen, transmission(30\%@330nm, 80\%@ $2.3 \mu \mathrm{m})$

- Master telescope pupil, transmission $(\approx 77 \%)$

- Adaptive optics Strehl ratio (30\% at $330 \mathrm{~nm})$, transmission

- Detector EMCCD, $\rho_{330} \approx 60 \%, \rho_{589} \approx 80 \%$

- Data processing phase restoration[7] or 3D cross correlations

Further improvements of our model will address the cone effect, the anisoplatism and the Na layer thickness.

From our model in the case of the $1.52 \mathrm{~m}$ at OHP, we predict a tilt Strehl ratio $\mathcal{S}_{\text {tilt }} \approx 30-35 \%$ at $\lambda \approx 550 \mathrm{~nm}$. 


\section{Construction of the demonstrator}

The ELP-OA demonstrator is being built at the OHP $1.52 \mathrm{~m}$ telescope. Figure 9 shows a schematic layout of the major components of the experiment.

The two laser chains locked at 589 and $569 \mathrm{~nm}$ are located at the ground level of the building, on basements independent from the telescope and its building. They are dye lasers, pulsed with a repetition rate of $10 \mathrm{kHz}$. They are pumped through fiber optics by 3 ETNA HP NdYAGs (Thalès). Then the 2 beams are transported inside tubes through the building to the dome. They enter the telescope through the North tip of the hour angle of its equatorial mount (or parallel to it). In such a way all incidence angles are kept constant as the telescope tracks.

Telescope vibrations are monitored with two pendular seismometers[8], one per telescope axis. The BOA-UV tip tilt mirror will be controlled both by the tip tilt signal from $\Delta \theta$ measurements (in close loop), and from the signal from the seismometers (in open loop).

Two auxiliary equipments are definitely required.

- A Generalized Seeing Monitor, on loan from Nice University thanks to A. Ziad and J. Borgnino, will be operated close to the telescope to get measurements of $r_{0}$ and $\tau_{0}$. They are inputs of the model, since they affect the BOA-UV Strehl ratio, and therefore the accuracy of $\Delta \theta$ measurements. Also, observations should not be carried out with conditions such as $d / r_{0} \gtrsim 1$. We have used a thermal imaging camera to evaluate the quality of the telescope environment, which clearly has to be improved.

- The column density of $\mathrm{Na}$ is a critical input of the model. Indeed it varies by a factor up to 6 along the year $[9,10]$. It will be monitored will from the OHP $1.2 \mathrm{~m}$ telescope, using a CCD camera and a filter or a grism.

We plan to have the first shoot at the sky (zenith) in July 2010 and to have the full ELP-OA experiment running in 2011

\section{Conclusion}

As a conclusion, we wish to mention a paragraph of the Analysis and roadmap from the E-ELT Adaptive Optics Working Group (2006) :

the polychromatic LGS development should be monitored and as for other novel concepts proposed in this document encouraged. Should the pending theoretical issues and simulations be positive, should be encouraged for on-sky demonstration.

We are now in the experimental work leading to on-sky demonstration.

\section{References}

1. Foy, R. and Labeyrie, A. AA 152, L29-32 (1985).

2. Pilkington, J. Nature 330, 116 (1987).

3. Filippenko, A. V. Pub. Astron. Soc. Pacific 94, 715-721 (1982).

4. Bellanger, V., Courcelle, A., and Petit, A. Computer Physics Comm. 162, 143-150 (2004).

5. Schöck, M., Foy, R., Pique, J.-P. et al. SPIE, 296-307, (2000).

6. Madec, P.-Y., Rabaud, D., Fleury, B., Conan, J.-M., L.Rousset-Rouvière, Mendez, F., Montri, J., Michau, V., Rousset, G., and Séchaud, M. La Lettre de l'OHP 16, 2-3 (1997).

7. Rondeau, X., Thiébaut, E., Tallon, M., and Foy, R. JOSA A 24 (2007).

8. Tokovinin, A. MNRAS 316, 637-641 August (2000).

9. Mégie, G., Bos, F., Blamont, J. E., and Chanin, M.-L. Planet. Space Sci. 26, 27-35 (1978).

10. Gardner, C., Voelz, D., Sechrist, C., and Segal, A. J. Geophys. Res 91, 13659-13673 (1986). 